\title{
Article
}

\section{Exponentiated transmuted lindley distribution with applications}

\author{
Emmanuel W. Okereke ${ }^{1, *}$ \\ 1 Department of Statistics, Michael Okpara University, Umudike, Nigeria. \\ * Correspondence: okereke.emmanuel@mouau.edu.ng \\ Received: 07 February 2019; Accepted: 29 May 2019; Published: 30 July 2019.
}

\begin{abstract}
In this paper, we study a new distribution called the exponentiated transmuted Lindley distribution. The proposed distribution has three special cases namely Lindley, exponentiated Lindley and transmuted Lindley distributions. Along with the basic properties of the distribution, the maximum likelihood technique of estimating the parameters of the distribution are discussed. Two applications of the distribution are also part of this article.
\end{abstract}

Keywords: Transmuted Lindley distribution, quantile function, skewness, kurtosis, maximum likelihood procedure.

MSC: 62N05, 90B25.

\section{Introduction}

$\mathbf{P}$ robability distributions are of paramount importance in many practical applications of statistics. In deed, several statistical methods are applicable when the data under consideration follow certain distributions. In a study, one may find a particular probability distribution useful in modelling a data set while in another study a different distribution may be required. The Lindley distribution is among the widely used distributions. It is mainly used to model lifetime data. Ghitany et al.[1] demonstrated the superiority of the Lindley distribution over the exponential distribution in modelling waiting time data of 100 customers in a bank. Another important application of the this distribution is the analysis of time series data. Popović et al. [2] introduced and showed the application of the autoregressive process of order one with Lindley marginal distribution.

Generalizations of several distributions are available in the statistical science literature. According to Fatima et al. [3], distributions are generalized so as to provide better fits to data and obtain more flexible models. Two common methods of generalizing distributions are the exponentiation method and quadratic rank transmutation map method. The generalized Lindley distribution introduced by Nadarajah et al. [4], was fitted to relief times of twenty patients and compared to Weibull, gamma, and lognormal distribution. The authors concluded that the generalized distribution provided better fits than the other three distributions. Merovci [5] introduced and determined properties of the transmuted Lindley distribution. His data analysis based on this distribution and remission times of 128 cancer patients showcased the superiority of the distribution over the exponential and Lindley distributions, which were all fitted to the data. Other Lindley-type distributions that have been fitted to the data include a new generalization of the transmuted Lindley distribution of Mansour et al.[6] and transmuted two-parameter Lindley distribution of Kemaloglu et al. [7]. According to Kemaloglu et al., the latter provided better fits to the data than any of Lindley and transmuted power Lindley distributions. Granzotto et al. [8] developed the transmuted power lindley distribution and showed that it can provide better fits to monthly rainfall data than the Lindley, power Lindley, weighted Lindley and transmuted Lindley distributions. In some situations, an exponentiated transmuted distribution, which is a generalization of a baseline distribution using both exponentiation and transmutation techniques outperforms its special cases [9-11]. On this note, we propose and study the properties of the exponentiated transmuted Lindley distribution (ETLD). Suppose there are $\alpha$ transmuted Lindley variables representing failure times of a component of a system, assumed to be independent. Another motivation for 
introducing the distribution is that it can be used to determine the probability that the system will fail before a given time. Again, the hazard rate function of the distribution is quite flexible, assuming various shapes, especially non monote shapes. Thus, the distribution will be of use in many practical applications of statistics.

\section{The proposed distribution}

From the work of Merovci [5], it is obvious that if a random variable $(X)$ has a transmuted Lindley distribution with parameters $\theta$ and $\lambda$, then its probability density function (pdf) is

$$
f_{1}(x)=\frac{\theta^{2}}{\theta+1}(1+x) e^{-\theta x}\left(1-\lambda+2 \lambda \frac{\theta+1+\theta x}{\theta+1} e^{-\theta x}\right), x>0, \theta>0,|\lambda| \leq 1
$$

Consequently, the cumulative distribution function (cdf) of the transmuted Lindley distributed random variable may be written as

$$
F_{1}(x)=\left(1-\frac{\theta+1+\theta x}{\theta+1} e^{-\theta x}\right)\left(1+\lambda \frac{\theta+1+\theta x}{\theta+1} e^{-\theta x}\right), x>0, \theta>0,|\lambda| \leq 1
$$

Given that $x>0, \alpha>0, \theta>0$ and $|\lambda| \leq 1$, the cdf of the exponentiated transmuted Lindley (ETL) distributed random variable $(X)$ becomes

$$
\begin{aligned}
F(x) & =\left(F_{1}(x)\right)^{\alpha} \\
& =\left[\left(1-\frac{\theta+1+\theta x}{\theta+1} e^{-\theta x}\right)\left(1+\lambda \frac{\theta+1+\theta x}{\theta+1} e^{-\theta x}\right)\right]^{\alpha}
\end{aligned}
$$

Using (3), the pdf of $X$ is found to be

$$
\begin{aligned}
f(x) & =\frac{\alpha \theta^{2}}{\theta+1}(1+x) e^{-\theta x}\left(1-\lambda+2 \lambda \frac{\theta+1+\theta x}{\theta+1} e^{-\theta x}\right) \\
& \times\left[\left(1-\frac{\theta+1+\theta x}{\theta+1} e^{-\theta x}\right)\left(1+\lambda \frac{\theta+1+\theta x}{\theta+1} e^{-\theta x}\right)\right]^{\alpha-1}
\end{aligned}
$$

The ETLD is actually a generalization of each of the Lindley, transmuted Lindley and exponentiated (generalized) Lindley distributions. When $\lambda=0$ and $\alpha=1$, the proposed distribution is basically a Lindley distribution. If $\alpha=1$, the distribution is the same as the transmuted Lindley distribution. The exponentiated Lindley distribution is a special of the ETL distribution for which $\lambda=0$. Figures 1 and 2 show that the pdf and cdf of an exponentiated transmuted Lindley distributed variable may have various shapes, depending on the values of $\theta, \lambda$ and $\alpha$.

\section{Quantile function}

The quantile function $x_{q}$ satisfies the equation

$$
\begin{aligned}
& F\left(x_{q}\right)=q \\
& \Rightarrow\left[\left(1-\frac{\theta+1+\theta x_{q}}{\theta+1} e^{-\theta x_{q}}\right)\left(1+\lambda \frac{\theta+1+\theta x_{q}}{\theta+1} e^{-\theta x_{q}}\right)\right]^{\alpha}=q
\end{aligned}
$$

From (5), we have

$$
\frac{\theta+1+\theta x_{q}}{\theta+1} e^{-\theta x_{q}}=\frac{\lambda-1+\sqrt{(1+\lambda)^{2}-4 \lambda q^{\frac{1}{\alpha}}}}{2 \lambda}
$$



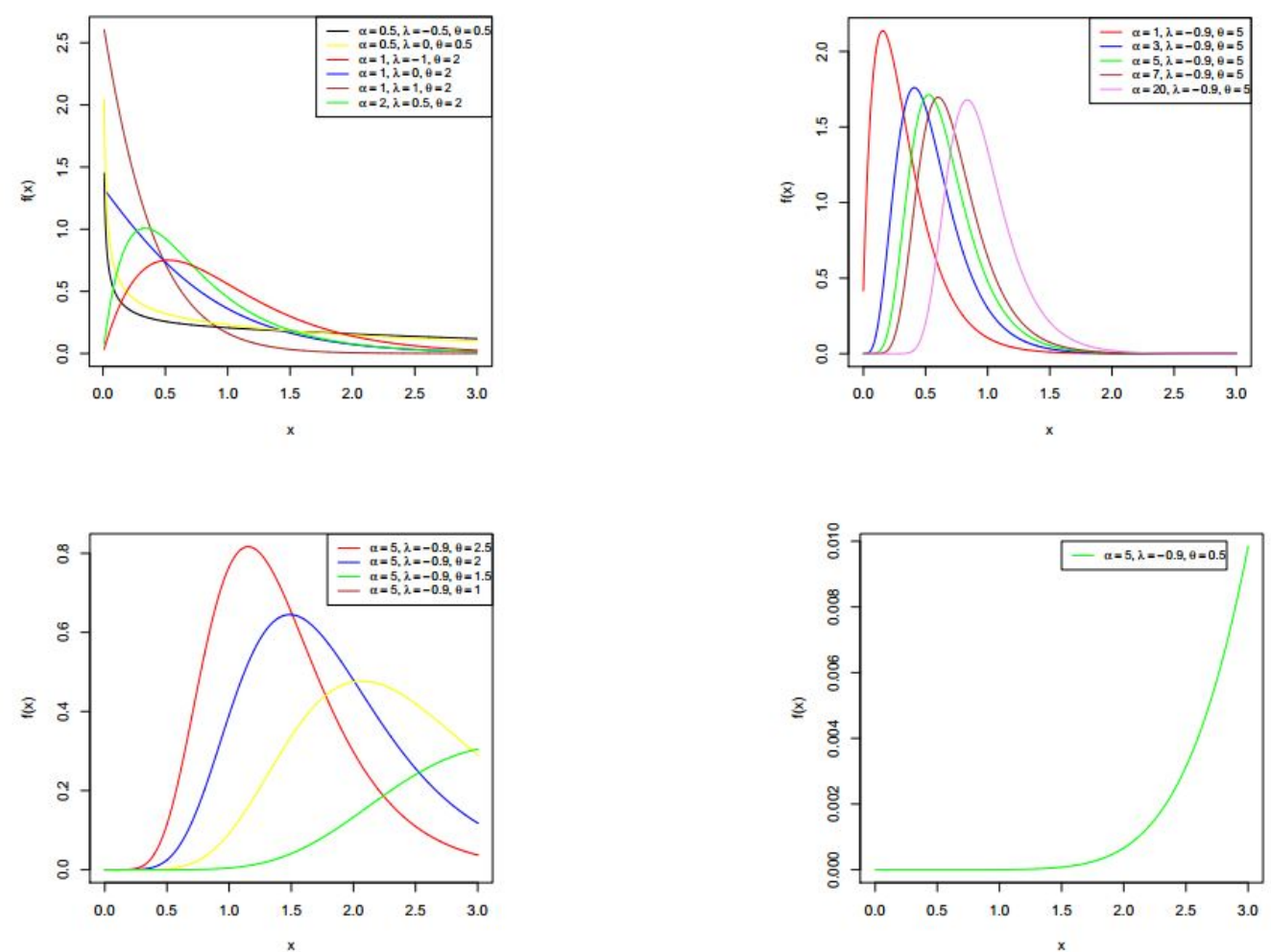

Figure 1. pdf of ETLD for selected values of $\alpha, \lambda$ and $\theta$

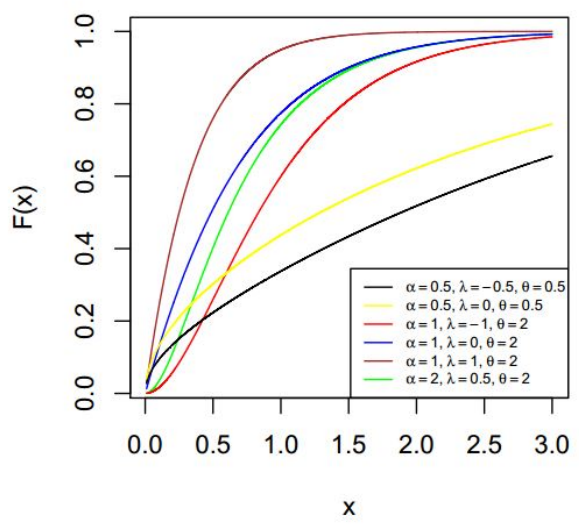

Figure 2. cdf of ETLD for selected values of $\alpha, \lambda$ and $\theta$

Applying the Lambert $W$ function and the method of Kemalogu et al. [7], we have the expression for $x_{q}$ as

$$
x_{q}=-\frac{\theta+1}{\theta}-\frac{1}{\theta} W_{-1}\left(-\left(\frac{\lambda-1+\sqrt{(1+\lambda)^{2}-4 \lambda q^{\frac{1}{\alpha}}}}{2 \lambda}\right) e^{-(\theta+1)}(\theta+1)\right) .
$$

In (7), $W_{-1}$ stands for the negative branch of the Lambert $W$ function. 


\section{Moments and moment generating function}

Given that $X$ is an $E T L$ random variable with parameters $\alpha, \lambda$ and $\theta$, then

$$
\begin{aligned}
E\left(X^{r}\right) & =\frac{\alpha \theta^{2}}{\theta+1} \int_{0}^{\infty} x^{r}(1+x) e^{-\theta x}\left(1-\lambda+2 \lambda \frac{\theta+1+\theta x}{\theta+1} e^{-\theta x}\right) \\
& \times\left[\left(1-\frac{\theta+1+\theta x}{\theta+1} e^{-\theta x}\right)\left(1+\lambda \frac{\theta+1+\theta x}{\theta+1} e^{-\theta x}\right)\right]^{\alpha-1} d x .
\end{aligned}
$$

To evaluate the raw moment, we may need the series representations

$$
\begin{gathered}
\left(1-\frac{\theta+1+\theta x}{\theta+1} e^{-\theta x}\right)^{\alpha-1}=\sum_{i=0}^{\infty}\left(\begin{array}{c}
\alpha-1 \\
i
\end{array}\right) \frac{(-1)^{i}}{(\theta+1)^{i}}(1+\theta(1+x))^{i} e^{-\theta i x}, \\
\left(1+\lambda \frac{\theta+1+\theta x}{\theta+1} e^{-\theta x}\right)^{\alpha-1}=\sum_{j=0}^{\infty}\left(\begin{array}{c}
\alpha-1 \\
j
\end{array}\right) \frac{\lambda^{j}}{(\theta+1)^{j}}(1+\theta(1+x))^{j} e^{-\theta j x}, \\
(1+\theta(1+x))^{i+j}=\sum_{k=0}^{i+j}\left(\begin{array}{c}
i+j \\
k
\end{array}\right) \theta^{k}(1+x)^{k}
\end{gathered}
$$

and

$$
(1+x)^{k+1}=\sum_{s=0}^{k+1}\left(\begin{array}{c}
k+1 \\
s
\end{array}\right) x^{s}
$$

Thus,

$$
\begin{aligned}
E\left(X^{r}\right) & =\frac{\alpha \theta^{2}}{\theta+1} \sum_{i=0}^{\infty} \sum_{j=0}^{\infty} \sum_{k=0}^{i+j} \sum_{s=0}^{k+1}\left(\begin{array}{c}
\alpha-1 \\
i
\end{array}\right)\left(\begin{array}{c}
\alpha-1 \\
j
\end{array}\right)\left(\begin{array}{c}
i+j \\
k
\end{array}\right)\left(\begin{array}{c}
k+1 \\
s
\end{array}\right) \\
& \times \frac{(-1)^{i} \lambda \theta^{j} \theta^{k}}{(\theta+1)^{i+j}} \int_{0}^{\infty} x^{r+s} e^{-\theta(i+j+1) x}\left(1-\lambda+2 \lambda \frac{\theta+1+\theta x}{\theta+1} e^{-\theta x}\right) d x
\end{aligned}
$$

The integral

$$
\begin{aligned}
& \int_{0}^{\infty} x^{r+s} e^{-\theta(i+j+1) x}\left(1-\lambda+2 \lambda \frac{\theta+1+\theta x}{\theta+1} e^{-\theta x}\right) d x \\
& =(1-\lambda) \int_{0}^{\infty} x^{r+s} e^{-\theta(i+j+1) x} d x+2 \lambda \int_{0}^{\infty} x^{r+s} e^{-\theta(i+j+2) x} d x+\frac{2 \lambda \theta}{\theta+1} \int_{0}^{\infty} x^{r+s+1} e^{-\theta(i+j+2) x} d x \\
& =\frac{(r+s) !}{\theta^{r+s+1}}\left(\frac{1-\lambda}{(i+j+1)^{r+s+1}}+\frac{2 \lambda}{(i+j+2)^{r+s+1}}+\frac{2 \lambda(r+s+1)}{(\theta+1)(i+j+2)^{r+s+2}}\right) .
\end{aligned}
$$

Using the results obtained above, the moment becomes

$$
\begin{aligned}
E\left(X^{r}\right) & =\frac{\alpha \theta^{2}}{1+\theta} \sum_{i=0}^{\infty} \sum_{j=0}^{\infty} \sum_{k=0}^{i+j} \sum_{s=0}^{k+1}\left(\begin{array}{c}
\alpha-1 \\
i
\end{array}\right)\left(\begin{array}{c}
\alpha-1 \\
j
\end{array}\right)\left(\begin{array}{c}
i+j \\
k
\end{array}\right)\left(\begin{array}{c}
k+1 \\
s
\end{array}\right) \\
& \times \frac{(-1)^{i} \lambda^{j} \theta^{k}(r+s) !}{(\theta+1)^{i+j} \theta^{r+s+1}}\left(\frac{1-\lambda}{(i+j+1)^{r+s+1}}+\frac{2 \lambda}{(i+j+2)^{r+s+1}}+\frac{2 \lambda(r+s+1)}{(\theta+1)(i+j+2)^{r+s+2}}\right) .
\end{aligned}
$$


The moment generating function of the ETL variable is

$$
\begin{aligned}
M_{X}(t) & =E\left(e^{t X}\right) \\
& =\sum_{\omega=0}^{\infty} \frac{t^{\omega} E\left(X^{\omega}\right)}{\omega !} \\
& =\frac{\alpha \theta^{2}}{1+\theta} \sum_{\omega=0}^{\infty} \sum_{i=0}^{\infty} \sum_{j=0}^{\infty} \sum_{k=0}^{i+j} \sum_{s=0}^{k+1}\left(\begin{array}{c}
\alpha-1 \\
i
\end{array}\right)\left(\begin{array}{c}
\alpha-1 \\
j
\end{array}\right)\left(\begin{array}{c}
i+j \\
k
\end{array}\right)\left(\begin{array}{c}
k+1 \\
s
\end{array}\right) \\
& \times \frac{(-1)^{i} \lambda^{j} \theta^{k}(\omega+s) ! t^{\omega}}{(\theta+1)^{i+j} \theta^{\omega+s+1} \omega !}\left(\frac{2 \lambda}{(i+j+1)^{\omega+s+1}}+\frac{2 \lambda(\omega+s+1)}{(i+j+2)^{\omega+s+1}}+\frac{1+j+2)^{\omega+s+2}}{(\theta+1)(i+j+j}\right) .
\end{aligned}
$$

With the formula for finding $E\left(X^{r}\right)$, the mean and variance of the $E T L$ distribution can be determined. The classical coefficients of skewness and kurtosis obtained using the rth raw moment formula have some weaknesses and are now replaced by the Bowley skewness (S) [12] and Moors kurtosis (K) [13] respectively. Consequently,

$$
S=\frac{Q\left(\frac{3}{4}\right)-2 Q\left(\frac{2}{4}\right)+Q\left(\frac{1}{4}\right)}{Q\left(\frac{3}{4}\right)-Q\left(\frac{1}{4}\right)}
$$

and

$$
K=\frac{Q\left(\frac{7}{8}\right)-Q\left(\frac{5}{8}\right)+Q\left(\frac{3}{8}\right)-Q\left(\frac{1}{8}\right)}{Q\left(\frac{6}{8}\right)-Q\left(\frac{2}{8}\right)}
$$

where $Q($.$) represents the quantile function.$

In Table 1, the mean, median, variance, Bowley skewness and Moors kurtosis are presented for various parameter values of the ETLD. We have computed these summary measures in order to examine the relationships between them and the parameters of the ETLD.

In addition to the numerical results in Table 1, we have provided plots (Figures 3 to 8 ) to ensure that we make a valid interpretation of the relationship between each of skewness $(S)$ and kurtosis $(K)$ and any of the parameters when the other two parameters are fixed. From Figures 3 and 4, we conclude that both $S$ and $K$ are nonincreasing functions of $\alpha$ if $\lambda$ and $\theta$ are fixed. Figures 5 and 6 show that as a function of $\lambda$ only, each of $S$ and $\mathrm{K}$ is unimodal. As shown in Figures 7 and $8, \mathrm{~S}$ and $\mathrm{K}$ are all nondecreasing functions of $\theta$ when $\alpha$ and $\lambda$ are fixed. If only $\alpha$ is held constant, Table 1 indicates that all of the mean, median and variance increase as $\lambda$ and $\theta$ jointly increase. Also, the variance increases provided each of the three parameters increases. Graphs of $\mathrm{S}$ and $\mathrm{K}$ against two parameters are displayed in Figures 9 to 14.

Though the results in Table 1 are primarily obtained to investigate some relationships, there also useful in examining whether the distribution is negatively skewed, symmetric or positively skewed. With the results, we can also determine if the distribution is platykurtic, mesokurtic or leptokurtic. With regard to the results, distribution is positively skewed and it can be platykurtic or leptokurtic, depending on the values of the parameters.

\section{Reliability analysis}

The reliability function refers to the probability that an item will not fail before a given time $t$. In terms of the exponentiated transmuted Lindley distribution with cdf $F(x)$, this function may be expressed as

$$
\begin{aligned}
R(x) & =1-F(x) \\
& =1-\left[\left(1-\frac{\theta+1+\theta x}{\theta+1} e^{-\theta x}\right)\left(1+\lambda \frac{\theta+1+\theta x}{\theta+1} e^{-\theta x}\right)\right]^{\alpha}
\end{aligned}
$$

Different shapes of the reliability function are graphically shown in Figure 3. In addition to the reliability function, one's desire may be to examine the hazard rate function defined by 
Table 1. Some Summary Measures based on ETLD

\begin{tabular}{|c|c|c|c|c|c|c|c|}
\hline$\alpha$ & $\lambda$ & $\theta$ & Mean & Median & Variance & S & K \\
\hline 0.1 & 0.2 & 0.5 & 0.5017 & 0.0049 & 1.9904 & 0.9637 & 4.2368 \\
\hline 0.5 & 0.2 & 0.5 & 1.9281 & 1.1229 & 8.9714 & 0.3213 & 0.8403 \\
\hline 0.9 & 0.2 & 0.5 & 2.8566 & 2.1517 & 14.8079 & 0.2156 & 0.5776 \\
\hline 1.3 & 0.2 & 0.5 & 3.5361 & 2.8890 & 19.8520 & 0.1817 & 0.4875 \\
\hline 1.7 & 0.2 & 0.5 & 4.0693 & 2.4577 & 24.3127 & 0.1648 & 0.4422 \\
\hline 2.1 & 0.2 & 0.5 & 4.5068 & 3.9192 & 28.3241 & 0.1544 & 0.4147 \\
\hline 2.5 & 0.2 & 0.5 & 4.8775 & 4.3073 & 31.9777 & 0.1473 & 0.3960 \\
\hline 2.9 & 0.2 & 0.5 & 5.1986 & 4.6417 & 35.3389 & 0.1422 & 0.3825 \\
\hline 0.5 & -0.9 & 2.5 & 0.1376 & 0.3455 & 0.1102 & 0.2429 & 0.6417 \\
\hline 0.5 & -0.5 & 2.5 & 0.1069 & 0.2474 & 0.0859 & 0.3036 & 0.7859 \\
\hline 0.5 & -0.1 & 2.5 & 0.0850 & 0.1721 & 0.0649 & 0.3835 & 0.9720 \\
\hline 0.5 & 0.1 & 2.5 & 0.0757 & 0.1451 & 0.0552 & 0.4070 & 1.0475 \\
\hline 0.5 & 0.5 & 2.5 & 0.0591 & 0.1076 & 0.0371 & 0.4198 & 1.1111 \\
\hline 0.5 & 0.9 & 2.5 & 0.0446 & 0.0841 & 0.0202 & 0.4083 & 1.0570 \\
\hline 2 & -0.1 & 0.1 & 27.4823 & 24.7747 & 978.2794 & 0.1305 & 0.3527 \\
\hline 2 & -0.1 & 0.5 & 4.9569 & 4.4027 & 33.2689 & 0.1351 & 0.3654 \\
\hline 2 & -0.1 & 0.9 & 2.5640 & 2.2480 & 9.1625 & 0.1418 & 0.3834 \\
\hline 2 & -0.1 & 1.3 & 1.6847 & 1.4618 & 4.0346 & 0.1482 & 0.4002 \\
\hline 2 & -0.1 & 1.7 & 1.2380 & 1.0656 & 2.2094 & 0.1535 & 0.4141 \\
\hline 2 & -0.1 & 2.1 & 0.9713 & 0.8307 & 1.3738 & 0.1579 & 0.4254 \\
\hline 2 & -0.1 & 2.5 & 0.7955 & 0.6770 & 0.9286 & 0.1613 & 0.4343 \\
\hline 2 & -0.1 & 2.9 & 0.6716 & 0.5694 & 0.6658 & 0.1640 & 0.4415 \\
\hline 0.1 & -0.9 & 2.5 & 0.1376 & 0.0051 & 0.1102 & 0.9178 & 2.6316 \\
\hline 0.5 & -0.5 & 2.5 & 0.4074 & 0.2474 & 0.3844 & 0.3036 & 0.7859 \\
\hline 0.9 & -0.1 & 2.5 & 0.5044 & 0.3590 & 0.4943 & 0.2447 & 0.6436 \\
\hline 1.3 & 0.1 & 2.5 & 0.5751 & 0.4395 & 0.5788 & 0.2179 & 0.5786 \\
\hline 1.7 & 0.5 & 2.5 & 0.5389 & 0.4154 & 0.4961 & 0.2201 & 0.5987 \\
\hline 2.1 & 0.9 & 2.5 & 0.4469 & 0.3651 & 0.3145 & 0.1845 & 0.5055 \\
\hline 0.1 & 0.2 & 0.5 & 0.5017 & 0.0049 & 0.9904 & 0.9637 & 4.2368 \\
\hline 0.5 & 0.2 & 1 & 0.8487 & 0.4440 & 1.8900 & 0.3716 & 0.9554 \\
\hline 0.9 & 0.2 & 1.5 & 0.7900 & 0.5455 & 1.2543 & 0.2611 & 0.6898 \\
\hline 1.3 & 0.2 & 2 & 0.7086 & 0.5384 & 0.8852 & 0.2218 & 0.5900 \\
\hline 1.7 & 0.2 & 2.5 & 0.6380 & 0.5089 & 0.6620 & 0.2011 & 0.5367 \\
\hline 2.1 & 0.2 & 3 & 0.5797 & 0.4765 & 0.5171 & 0.1880 & 0.5029 \\
\hline 0.5 & -0.9 & 0.5 & 3.1806 & 2.4980 & 17.7535 & 0.1827 & 0.4921 \\
\hline 0.5 & -0.5 & 1 & 1.1941 & 0.7879 & 3.0667 & 0.2598 & 0.6845 \\
\hline 0.5 & -0.1 & 1.5 & 0.6118 & 0.3299 & 0.9479 & 0.3631 & 0.9160 \\
\hline 0.5 & 0.1 & 2 & 0.3921 & 0.1925 & 0.4225 & 0.3999 & 1.0253 \\
\hline 0.5 & 0.5 & 2.5 & 0.2379 & 0.1076 & 0.1723 & 0.4198 & 1.1112 \\
\hline 0.5 & 0.9 & 3 & 0.1433 & 0.0670 & 0.0612 & 0.4113 & 1.0684 \\
\hline 0.1 & -0.9 & 0.5 & 0.9383 & 0.0535 & 4.2448 & 0.8979 & 2.1445 \\
\hline 0.5 & -0.5 & 1 & 1.1941 & 0.7879 & 3.0667 & 0.2598 & 0.6845 \\
\hline 0.9 & -0.1 & 1.5 & 0.9154 & 0.6720 & 1.5723 & 0.2271 & 0.5999 \\
\hline 1.3 & 0.1 & 2 & 0.7450 & 0.5752 & 0.9587 & 0.2118 & 0.5629 \\
\hline 1.7 & 0.5 & 2.5 & 0.5389 & 0.4154 & 0.41961 & 0.2201 & 0.5987 \\
\hline 2.1 & 0.9 & 3 & 0.3609 & 0.2933 & 0.2068 & 0.1878 & 0.5147 \\
\hline
\end{tabular}

$$
h(x)=\frac{f(x)}{R(x)}
$$

where $f(x)$ and $R(x)$ are defined in (4) and (9) respectively. Figure 4 is indicative of the effect of parameter value combinations on the shape of the hazard rate function of the ETLD. 


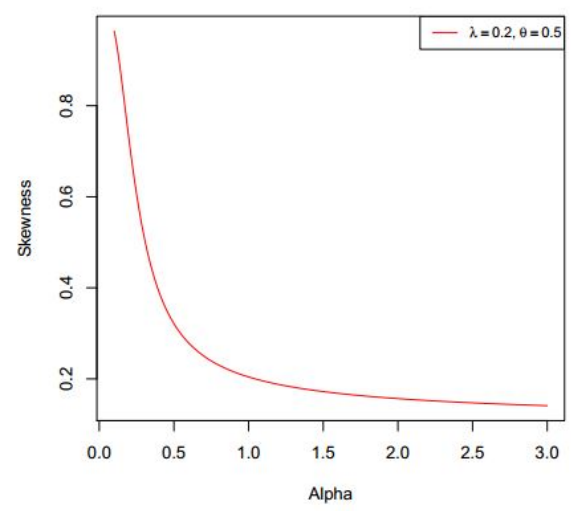

Figure 3. Skewness of ETLD when $\lambda=0.2$ and $\theta=0.5$

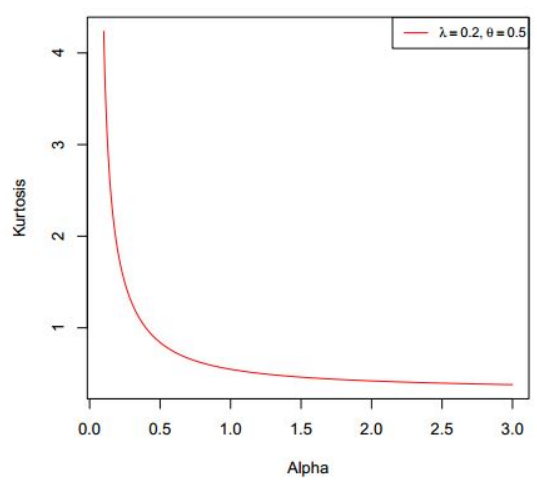

Figure 4. Kurtosis of ETLD when $\lambda=0.2$ and $\theta=0.5$

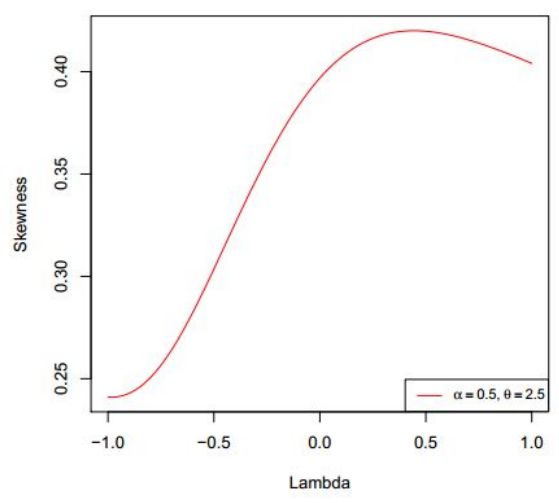

Figure 5. Skewness of ETLD for $\alpha=0.5$ and $\theta=2.5$

\section{Rényi entropy}

The concept of entropy is being used to describe the amount of uncertainty in a random variable. A popular measure of entropy is the Rényi entropy, which, for an ETL variable, is defined as

$$
I_{R}(\delta)=\frac{1}{1-\delta} \log \int_{0}^{\infty} f^{\delta}(x) d x, \delta>0, \delta \neq 1
$$




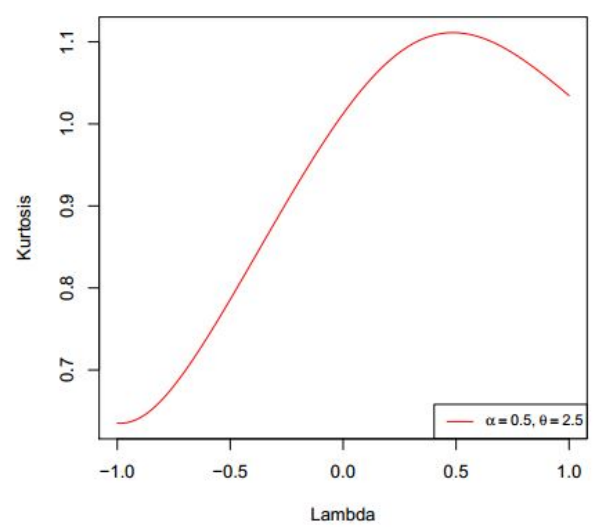

Figure 6. Kurtosis of ETL distribution $\alpha=0.5$ and $\theta=2.5$

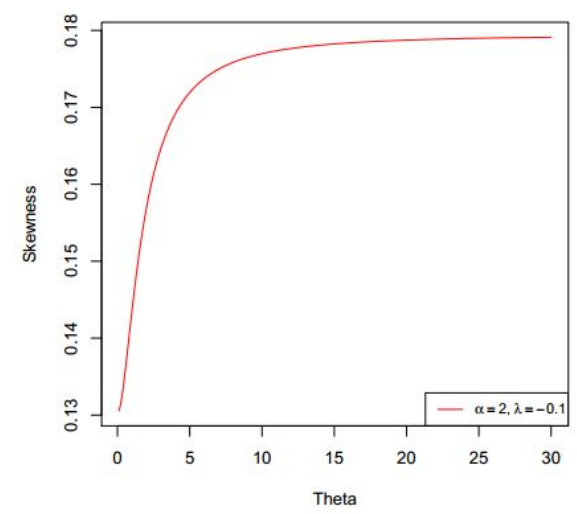

Figure 7. Skewness of ETLD for $\alpha=2$ and $\lambda=-0.1$

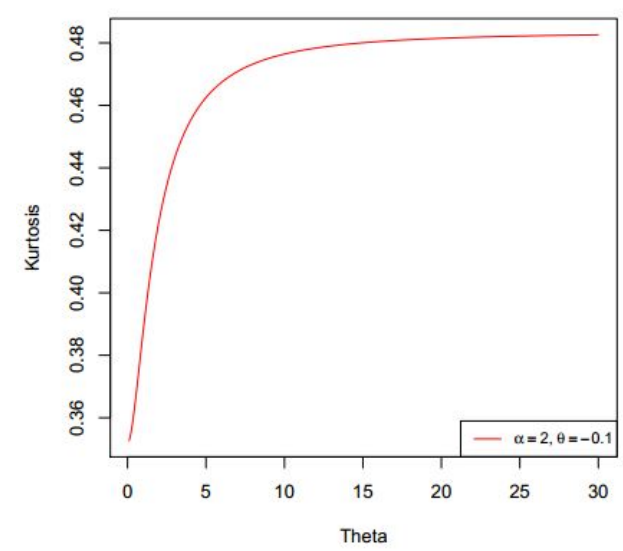

Figure 8. Kurtosis of ETLD for $\alpha=2$ and $\lambda=-0.1$

After substituting into (11) the expression for $f(x)$ as stated in (4), the following result is obtained:

$$
I_{R}(\delta)=\frac{1}{1-\delta} \log \int_{0}^{\infty}\left(\begin{array}{c}
\frac{\alpha \theta^{2}}{1+\theta}(1+x) e^{-\theta x}\left(1-\lambda+2 \lambda \frac{\theta+1+\theta x}{\theta+1} e^{-\theta x}\right) \\
\times\left[\left(1-\frac{\theta+1+\theta x}{\theta+1} e^{-\theta x}\right)\left(1+\lambda \frac{\theta+1+\theta x}{\theta+1} e^{-\theta x}\right)\right]^{\alpha-1}
\end{array}\right)^{\delta} .
$$




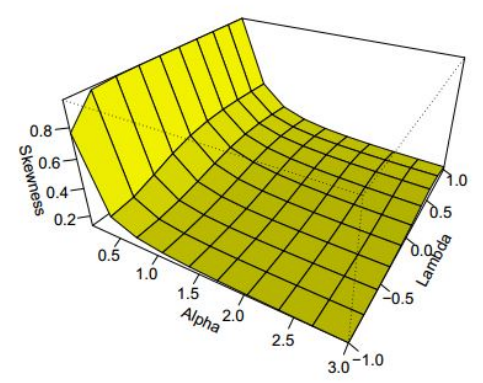

Figure 9. Skewness of ETLD when $\theta=2.5$

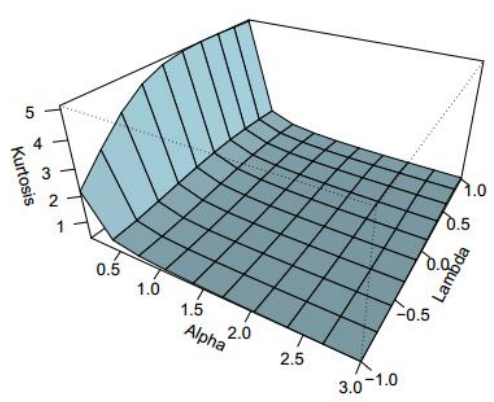

Figure 10. Kurtosis of ETLD when $\theta=2.5$

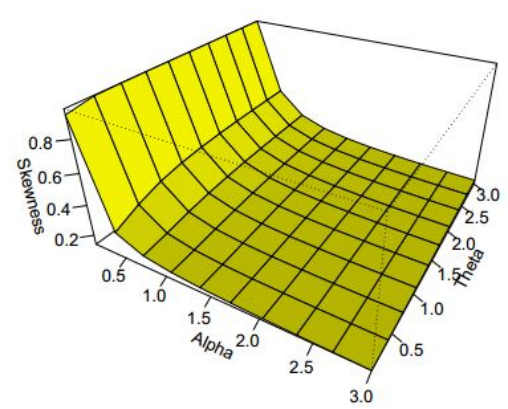

Figure 11. Skewness of $E T L D$ for $\lambda=0.2$

To evaluate (12), we use the series representations:

$$
\begin{gathered}
\left(1-\lambda+2 \lambda \frac{\theta+1+\theta x}{\theta+1} e^{-\theta x}\right)^{\delta}=(1-\lambda)^{\delta} \sum_{a=0}^{\infty}\left(\begin{array}{c}
\delta \\
a
\end{array}\right)\left(\frac{2 \lambda}{1-\lambda}\right)^{a}\left(1+\frac{\theta x}{\theta+1}\right)^{a} e^{-a \theta x} \\
\left(1-\left(1+\frac{\theta x}{\theta+1}\right) e^{-\theta x}\right)^{(\alpha-1) \delta}=\sum_{b=0}^{\infty}\left(\begin{array}{c}
(\alpha-1) \delta \\
b
\end{array}\right)(-1)^{b}\left(1+\frac{\theta x}{\theta+1}\right)^{b} e^{-b \theta x} \\
\left(1+\lambda\left(1+\frac{\theta x}{\theta+1}\right) e^{-\theta x}\right)^{(\alpha-1) \delta}=\sum_{c=0}^{\infty}\left(\begin{array}{c}
(\alpha-1) \delta \\
c
\end{array}\right) \lambda^{c}\left(1+\frac{\theta x}{\theta+1}\right)^{c} e^{-c \theta x}
\end{gathered}
$$




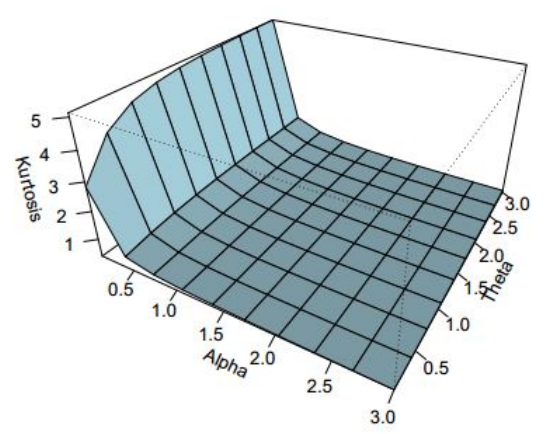

Figure 12. Kurtosis of $E T L D$ for $\lambda=0.2$

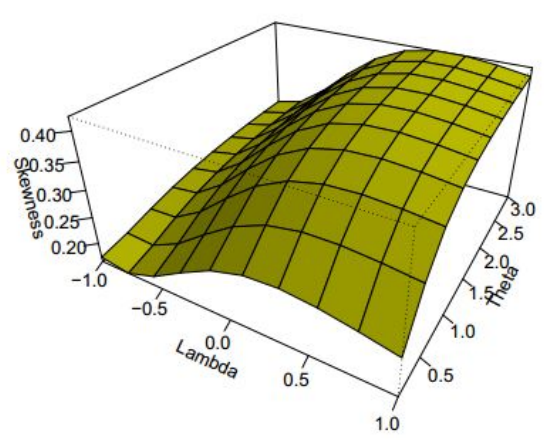

Figure 13. Skewness of ETLD for $\alpha=0.5$

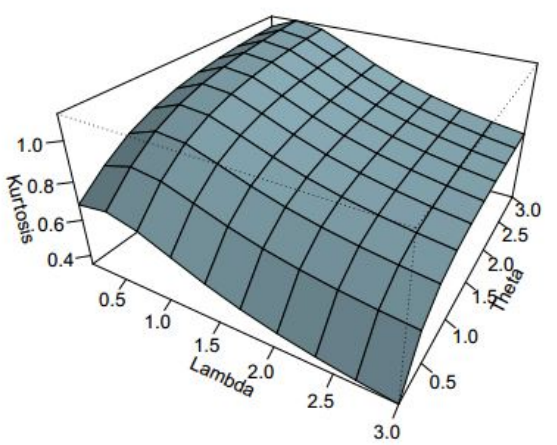

Figure 14. Kurtosis of $E T L D$ for $\alpha=0.5$

As a consequence, we obtain 


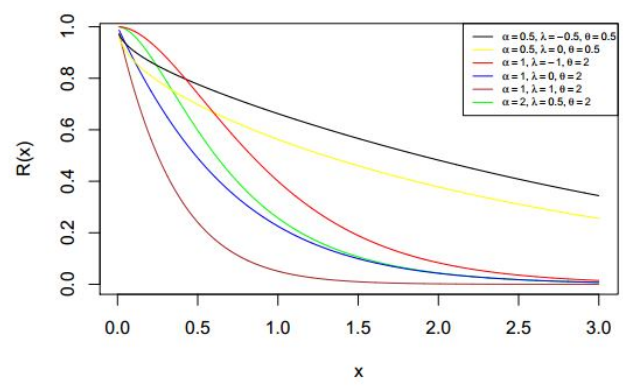

Figure 15. Kurtosis of ETLD for $\alpha=0.5$

$$
I_{R}(\delta)=\frac{1}{1-\delta} \log \int_{0}^{\infty}\left(\begin{array}{c}
\left(\frac{\alpha \theta^{2}}{1+\theta}\right)^{\delta}(x+1)^{\delta}(1-\lambda)^{\delta} \sum_{a=0}^{\infty} \sum_{b=0}^{\infty} \sum_{c=0}^{\infty} \\
a
\end{array}\right)\left(\begin{array}{c}
(\alpha-1) \delta \\
b \\
\frac{(1+\theta(x+1))^{a+b+c}}{(\theta+1)^{a+b+c}} e^{-(a+b+c+\delta) \theta x}\left(\begin{array}{c}
(\alpha-1) \delta \\
c
\end{array}\right)\left(\frac{2 \lambda}{1-\lambda}\right)^{a}(-1)^{b} \lambda^{c}
\end{array}\right) d x
$$

With $(1+\theta(x+1))^{a+b+c}=\sum_{d=0}^{a+b+c}\left(\begin{array}{c}a+b+c \\ d\end{array}\right) \theta^{d}(x+1)^{d}$ and $(x+1)^{d+\delta}=\sum_{u=0}^{\infty}\left(\begin{array}{c}d+\delta \\ u\end{array}\right) x^{u},(13)$ can be written as

$$
\begin{aligned}
& I_{R}(\delta)=\frac{1}{1-\delta} \log \left(\begin{array}{c}
\left(\frac{\alpha \theta^{2}}{1+\theta}\right)^{\delta}(1-\lambda)^{\delta} \sum_{a=0}^{\infty} \sum_{b=0}^{\infty} \sum_{c=0}^{\infty} \sum_{d=0}^{a+b+c} \sum_{u=0}^{\infty} \\
a \\
\left(\begin{array}{c}
(\alpha-1) \delta \\
b
\end{array}\right)\left(\begin{array}{c}
(\alpha-1) \delta \\
c
\end{array}\right)\left(\begin{array}{c}
a+b+c \\
d
\end{array}\right)\left(\begin{array}{c}
d+\delta \\
u
\end{array}\right) \\
\left(\frac{2 \lambda}{1-\lambda}\right)^{a} \frac{(-1)^{b} \lambda^{c} \theta^{d}}{(\theta+1)^{a+b+c}} \int_{0}^{\infty} x^{u} e^{-(a+b+c+\delta) \theta x} d x
\end{array}\right) \\
& =\frac{\delta}{1-\delta} \log \alpha+\frac{2 \delta}{1-\delta} \log \theta-\frac{\delta}{1-\delta} \log (\theta+1)+\frac{\delta}{1-\delta} \log (1-\lambda) \\
& +\frac{1}{1-\delta} \log \left(\left(\begin{array}{c}
\delta \\
a
\end{array}\right)\left(\begin{array}{c}
(\alpha-1) \delta \\
b \\
\left(\frac{2 \lambda}{1-\lambda}\right)^{a} \frac{(-1}{(\theta+1)^{a+b+c} \theta^{u+1}(a+b+c+\delta)^{u+1}} \\
c
\end{array} \sum_{b=0}^{\infty} \sum_{c=0}^{\infty} \sum_{d=0}^{a+b+c} \sum_{u=0}^{\infty}\left(\begin{array}{c}
a+b+c \\
d
\end{array}\right)\left(\begin{array}{c}
d+\delta \\
u
\end{array}\right)\right),\right.
\end{aligned}
$$

where

$$
\int_{0}^{\infty} x^{u} e^{-(a+b+c+\delta) \theta x} d x=\frac{u !}{\theta^{u+1}(a+b+c+\delta)^{u+1}}
$$

\section{Estimation}

The likelihood function of a random sample from the ETLD with parameters $\theta, \lambda$ and $\alpha$ is

$$
\begin{aligned}
L & =\prod_{i=1}^{n} f\left(x_{i} ; \alpha, \lambda, \theta\right) \\
& =\left(\frac{\alpha \theta^{2}}{\theta+1}\right)^{n} \times \prod_{i=1}^{n}\left(\begin{array}{c}
\left(1+x_{i}\right) e^{-\theta x_{i}}\left(1-\lambda+2 \lambda \frac{\theta+1+\theta x_{i}}{\theta+1} e^{-\theta x_{i}}\right) \\
\times\left[\left(1-\frac{\theta+1+\theta x_{i}}{\theta+1} e^{-\theta x_{i}}\right)\left(1+\lambda \frac{\theta+1+\theta x_{i}}{\theta+1} e^{-\theta x_{i}}\right)\right]^{\alpha-1}
\end{array}\right)
\end{aligned}
$$



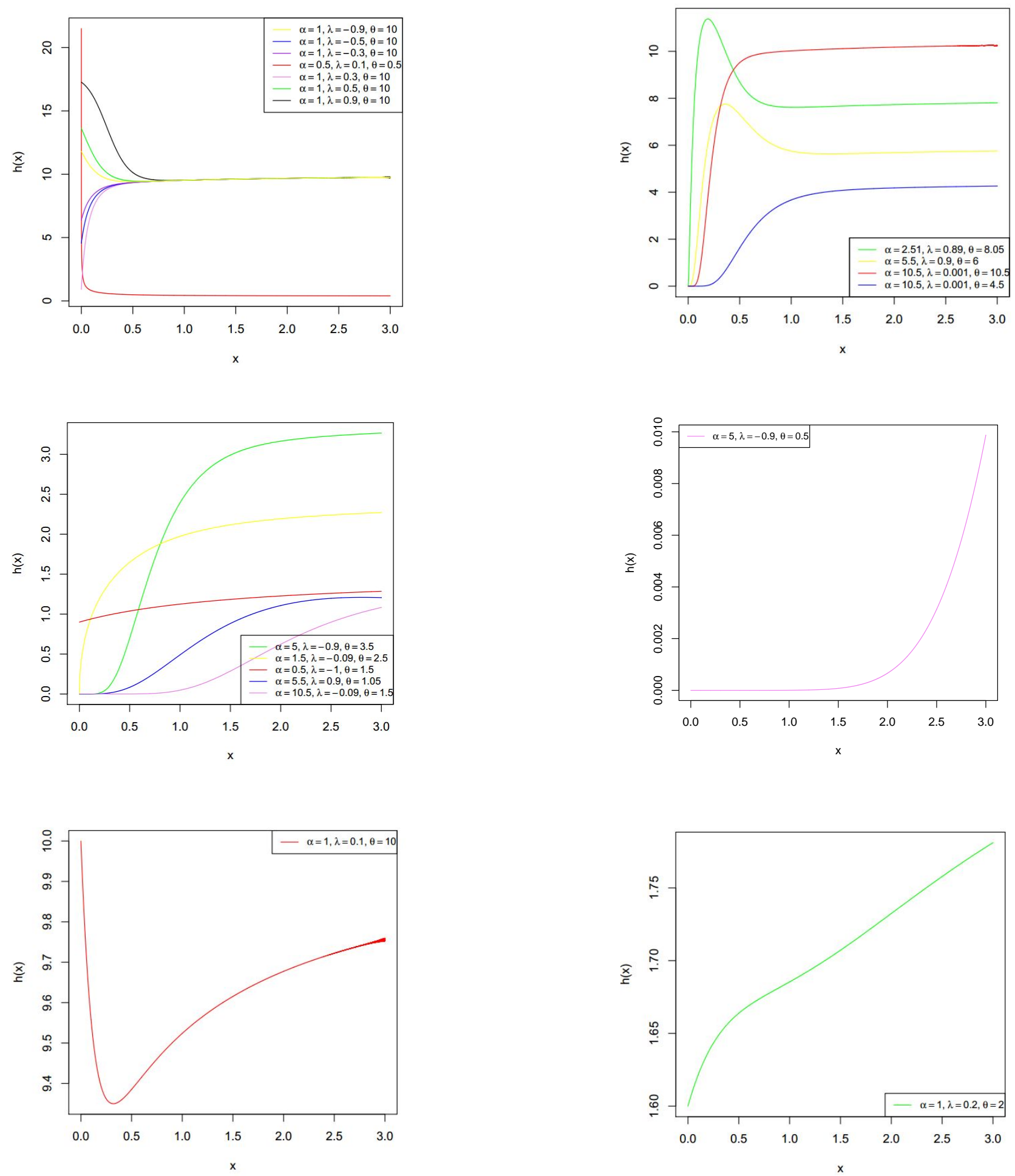

Figure 16. Hazard rate function of ETLD for selected values of $\alpha, \beta$ and $\theta$

The log-likelihood function can be written as

$$
\begin{aligned}
\ln L & =n \ln \alpha+2 n \ln \theta-n \ln (\theta+1)+\sum_{i=1}^{n} \ln \left(x_{i}+1\right)-\theta \sum_{i=1}^{n} x_{i} \\
& +\sum_{i=1}^{n} \ln \left(1-\lambda+2 \lambda \frac{\theta+1+\theta x_{i}}{\theta+1} e^{-\theta x_{i}}\right)+(\alpha-1) \sum_{i=1}^{n} \ln \left(1-\frac{\theta+1+\theta x_{i}}{\theta+1} e^{-\theta x_{i}}\right) \\
& +(\alpha-1) \sum_{i=1}^{n} \ln \left(1+\lambda \frac{\theta+1+\theta x_{i}}{\theta+1} e^{-\theta x_{i}}\right) .
\end{aligned}
$$


Maximum likelihood estimates (MLEs) of the three parameters $\theta, \lambda$ and $\alpha$ are determined by solving simultaneously the equations $\frac{\partial \ln L}{\partial \alpha}=0, \frac{\partial \ln L}{\partial \lambda}=0$ and $\frac{\partial \ln L}{\partial \theta}=0$. Consequently, the equations $\frac{\partial \ln L}{\partial \alpha}=0, \frac{\partial \ln L}{\partial \lambda}=0$ and $\frac{\partial \ln L}{\partial \theta}=0$, are respectively given as

$$
\begin{aligned}
& \frac{n}{\alpha}+\sum_{i=1}^{n} \ln \left(1-\frac{\theta+1+\theta x_{i}}{\theta+1} e^{-\theta x_{i}}\right)+\sum_{i=1}^{n} \ln \left(1+\lambda \frac{\theta+1+\theta x_{i}}{\theta+1} e^{-\theta x_{i}}\right)=0 ; \\
& \sum_{i=1}^{n} \frac{2\left(\frac{\theta+1+\theta x_{i}}{\theta+1}\right) e^{-\theta x_{i}}-1}{1-\lambda+2 \lambda \frac{\theta+1+\theta x_{i}}{\theta+1} e^{-\theta x_{i}}}+(\alpha-1) \sum_{i=1}^{n} \frac{\left(\frac{\theta+1+\theta x_{i}}{\theta+1}\right) e^{-\theta x_{i}}}{1+\lambda \frac{\theta+1+\theta x_{i}}{\theta+1} e^{-\theta x_{i}}}=0 ; \\
& \frac{2 n}{\theta}-\frac{n}{\theta+1}-\sum_{i=1}^{n} x_{i}-2 \lambda \theta \sum_{i=1}^{n} \frac{\left(\theta x_{i}+x_{i}+\theta+2\right) x_{i} e^{-\theta x_{i}}}{(\theta+1)^{2}\left(1-\lambda+2 \lambda \frac{\theta+1+\theta x_{i}}{\theta+1} e^{-\theta x_{i}}\right)} \\
& +\theta(\alpha-1) \sum_{i=1}^{n} \frac{\left(\theta x_{i}+x_{i}+\theta+2\right) x_{i} e^{-\theta x_{i}}}{(\theta+1)^{2}\left(1-\frac{\theta+1+\theta x_{i}}{\theta+1} e^{-\theta x_{i}}\right)} \\
& -\lambda \theta(\alpha-1) \sum_{i=1}^{n} \frac{\left(\theta x_{i}+x_{i}+\theta+2\right) x_{i} e^{-\theta x_{i}}}{(\theta+1)^{2}\left(1+\lambda \frac{\theta+1+\theta x_{i}}{\theta+1} e^{-\theta x_{i}}\right)}=0 .
\end{aligned}
$$

Closed form solution of (15), (16) and (17) cannot be obtained. A numerical approach based on quasi-Newton algorithm may then be used to solve these equations. Interval estimation of any of the parameters of the ETL distribution is possible when the necessary standard error estimate is known. As $n \rightarrow \infty$, the MLE $\hat{\gamma}=(\hat{\alpha}, \hat{\lambda}, \hat{\theta})^{\prime}$ of $\gamma=(\alpha, \lambda, \theta)^{\prime}$ is asymptotically normally distributed with mean $\gamma$ and variance-covariance matrix

$$
V=\left(\begin{array}{lll}
V_{11} & V_{12} & V_{13} \\
V_{21} & V_{22} & V_{23} \\
V_{31} & V_{32} & V_{33}
\end{array}\right)=\left(\begin{array}{lll}
B_{11} & B_{12} & B_{13} \\
B_{21} & B_{22} & B_{23} \\
B_{31} & B_{32} & B_{33}
\end{array}\right)^{-1},
$$

where

$$
\begin{gathered}
B_{11}=-\frac{\partial^{2} \ln L}{\partial \alpha^{2}}, B_{12}=-\frac{\partial^{2} \ln L}{\partial \alpha \partial \lambda}, B_{13}=-\frac{\partial^{2} \ln L}{\partial \alpha \partial \theta} \\
B_{22}=-\frac{\partial^{2} \ln L}{\partial \lambda^{2}}, B_{23}=-\frac{\partial^{2} \ln L}{\partial \lambda \partial \theta} \text { and } B_{33}=-\frac{\partial^{2} \ln L}{\partial \theta^{2}} .
\end{gathered}
$$

Hence, approximate $100(1-\eta) \%$ confidence intervals for $\alpha, \lambda$ and $\theta$ are $\hat{\alpha} \pm z_{\frac{\eta}{2}} \sqrt{\hat{V}_{11}}, \hat{\lambda} \pm z_{\frac{\eta}{2}} \sqrt{\hat{V}_{22}}$ and $\hat{\theta} \pm$ $z_{\frac{\eta}{2}} \sqrt{\hat{V}_{33}}$ respectively, where $z_{\frac{\eta}{2}}$ is the upper $\frac{\eta}{2}$ th percentile of the standard normal distribution. The R package is useful in obtaining the parameter estimates and their associated standard errors.

\section{Order statistics}

Consider a random sample $X_{1}, X_{2}, \cdots, X_{n}$ from the ETL distribution with pdf $f(x)$ and $\operatorname{cdf} F(x)$. Let $X_{(1)}, X_{(2)}, \cdots, X_{(n)}$ be the corresponding order statistics. The pdf of the $k$ th order statistic $X_{(k)}$ can be written as

$$
\begin{aligned}
f_{X_{(k)}}(x) & =\frac{n !}{(k-1) !(n-k) !} f(x)(F(x))^{k-1}(1-F(x))^{n-k} \\
& =\frac{\alpha \theta^{2} n !}{(\theta+1)(k-1) !(n-k) !}(1+x) e^{-\theta x}\left(1-\lambda+2 \lambda \frac{\theta+1+\theta x}{\theta+1} e^{-\theta x}\right) \\
& \times\left(\left(1-\frac{\theta+1+\theta x}{\theta+1} e^{-\theta x}\right)\left(1+\lambda \frac{\theta+1+\theta x}{\theta+1} e^{-\theta x}\right)\right)^{\alpha-1} \\
& \times\left(\left(1-\frac{\theta+1+\theta x}{\theta+1} e^{-\theta x}\right)\left(1+\lambda \frac{\theta+1+\theta x}{\theta+1} e^{-\theta x}\right)\right)^{\alpha(k-1)} \\
& \times\left(1-\left(\left(1-\frac{\theta+1+\theta x}{\theta+1} e^{-\theta x}\right)\left(1+\lambda \frac{\theta+1+\theta x}{\theta+1} e^{-\theta x}\right)\right)^{\alpha}\right)^{n-k} .
\end{aligned}
$$


To simplify the expression for $f_{X_{(k)}}(x)$ in the equation above, we employ the binomial expansion

$$
\begin{aligned}
& \left(1-\left(\left(1-\frac{\theta+1+\theta x}{\theta+1} e^{-\theta x}\right)\left(1+\lambda \frac{\theta+1+\theta x}{\theta+1} e^{-\theta x}\right)\right)^{\alpha}\right)^{n-k} \\
= & \sum_{\omega=0}^{n-k}(-1)^{\omega}\left(\begin{array}{c}
n-k \\
\omega
\end{array}\right)\left(1-\frac{\theta+1+\theta x}{\theta+1} e^{-\theta x}\right)^{\alpha \omega}\left(1+\lambda \frac{\theta+1+\theta x}{\theta+1} e^{-\theta x}\right)^{\alpha \omega} .
\end{aligned}
$$

Thus,

$$
\begin{aligned}
f_{X_{(k)}}(x) & =\frac{\alpha \theta^{2} n !}{(\theta+1)(k-1) !(n-k) !}(1+x) e^{-\theta x}\left(1-\lambda+2 \lambda \frac{\theta+1+\theta x}{\theta+1} e^{-\theta x}\right) \\
& \times \sum_{\omega=0}^{n-k}(-1)^{\omega}\left(\begin{array}{c}
n-k \\
\omega
\end{array}\right)\left(1-\frac{\theta+1+\theta x}{\theta+1} e^{-\theta x}\right)^{\alpha(\omega+k)-1}\left(1+\lambda \frac{\theta+1+\theta x}{\theta+1} e^{-\theta x}\right)^{\alpha(\omega+k)-1} .
\end{aligned}
$$

If $k=1$, we have the pdf of the first order statistic $\left(X_{(1)}\right)$ defined by

$$
\begin{aligned}
f_{X_{(1)}}(x) & =\frac{\alpha \theta^{2} n}{(\theta+1)}(1+x) e^{-\theta x}\left(1-\lambda+2 \lambda \frac{\theta+1+\theta x}{\theta+1} e^{-\theta x}\right) \\
& \times \sum_{\omega=0}^{n-1}(-1)^{\omega}\left(\begin{array}{c}
n-k \\
\omega
\end{array}\right)\left(1-\frac{\theta+1+\theta x}{\theta+1} e^{-\theta x}\right)^{\alpha(\omega+1)-1}\left(1+\lambda \frac{\theta+1+\theta x}{\theta+1} e^{-\theta x}\right)^{\alpha(\omega+1)-1} .
\end{aligned}
$$

Similarly, the pdf of the $n t h$ order statistic $\left(X_{(n)}\right)$ is

$$
\begin{aligned}
f_{X_{(n)}}(x) & =\frac{\alpha \theta^{2} n}{(\theta+1)}(1+x) e^{-\theta x}\left(1-\lambda+2 \lambda \frac{\theta+1+\theta x}{\theta+1} e^{-\theta x}\right) \\
& \times\left(1-\frac{\theta+1+\theta x}{\theta+1} e^{-\theta x}\right)^{\alpha(\omega+n)-1}\left(1+\lambda \frac{\theta+1+\theta x}{\theta+1} e^{-\theta x}\right)^{\alpha(\omega+n)-1} .
\end{aligned}
$$

\section{Application of the $E T L D$}

In this section, the usefulness of the ETLD is illustrated using two real data sets. The first data, originally presented by Smithson et al. [14], pertain to a study on anxiety performed in a group of 166 "normal "women outside of a pathological clinical picture (Townsville, Queensland, Australia). The data have been used for a numerical illustration by Bourguignon et al. [15]. They are as follows: $0.01,0.17,0.01,0.05,0.09,0.41,0.05,0.01$, $0.13,0.01,0.05,0.17,0.01,0.09,0.01,0.05,0.09,0.09,0.05,0.01,0.01,0.01,0.29,0.01,0.01,0.01,0.01,0.01,0.01,0.01$, $0.01,0.09,0.37,0.05,0.01,0.05,0.29,0.09,0.01,0.25,0.01,0.09,0.01,0.05,0.21,0.01,0.01,0.01,0.13,0.17,0.37,0.01$, $0.01,0.09,0.57,0.01,0.01,0.13,0.05,0.01,0.01,0.01,0.01,0.09,0.13,0.01,0.01,0.09,0.09,0.37,0.01,0.05,0.01,0.01$ ， $0.13,0.01,0.57,0.01,0.01,0.09,0.01,0.01,0.01,0.01,0.01,0.01,0.05,0.01,0.01,0.01,0.13,0.01,0.25,0.01,0.01,0.09$, $0.13,0.01,0.01,0.05,0.13,0.01,0.09,0.01,0.05,0.01,0.05,0.01,0.09,0.01,0.37,0.25,0.05,0.05,0.25,0.05,0.05$, $0.01,0.05,0.01,0.01,0.01,0.17,0.29,0.57,0.01,0.05,0.01,0.09,0.01,0.09,0.49,0.45,0.01,0.01,0.01,0.05,0.01,0.17$, $0.01,0.13,0.01,0.21,0.13,0.01,0.01,0.17,0.01,0.01,0.21,0.13,0.69,0.25,0.01,0.01,0.09,0.13,0.01,0.05,0.01,0.01$ ， $0.29,0.25,0.49,0.01,0.01$.

The second data set comprising the salaries (in dollars) of 818 professional baseball players for the 2008 are reported by Oluyede et al. [16] and are as follows:

$0.403,1.75,6,0.4345,4.956237,5.5,0.75,0.475,1.5,11.666666,13,0.4186,2.5,0.406,13.1,1.775,2.7875$, $0.4,0.8,6.25,0.404,1,1.0325,0.42245,9,4.05,0.4,8.75,1.75,5.9,1.75,4,0.4551,3.125,0.975,5.5,1.5,5,1.5,1.7$ $10,15,0.4073,1.4,8,6.25,0.441,3.65,2,0.800002,33,0.4,1.98125,0.424,0.5,1.5,0.4363,3.5,1,15.285714,1.25$, $3.666666,0.75,0.401,5.5,0.4142,0.4275,0.403,5.4,0.4115,7,0.4,7.5,0.4,1.95,19,0.4,20.625,0.5,0.675,0.452,3.05$, $5,4.766666,5.5,7,0.432975,0.4044,8.25,0.445,3.5,4,0.4275,0.75,0.414,5,0.4324,0.4333,2.8,0.425,6.25,10,2.3$, $6.925,0.4,0.4309,1.255,0.475,0.7125,7,10,0.75,4.65,0.4,0.4,0.4337,0.425,0.43,1.1,7.05,14,3.25,0.405,0.41,0.75$ , 0.5,0.4115, $9,0.415,0.439,6,0.41,11,3.25,2.95,2.535,0.43,1.625,0.61,0.95,0.41,0.675,0.4104,5,2.525,12.5,1.055$, 
$1.5,5,4,1.6875,1.000018,0.4,0.4225,0.8,9.375,2.6,4.75,0.4,0.4,6,3.325,0.4,4.2,0.75,0.449,1.6625,0.42,0.4005$, $0.55,1.45,0.4215,2,0.5,0.4,11.5,4.625,2.1,3.6,7,2,14.811414,0.4,0.4,3.5,1.3,0.4225,1.625,0.401,8,0.426,5.3$, $1,1.29,2,0.475,3.75,0.425,4.25,2.8,0.404,8,5,0.4075,0.8,0.4245,0.415,0.41,2.9,4.445,0.412,3,5.5,1.2,0.4075$, $0.41,0.40175,0.4028,6.5,13.25,16.6,1.4,12.75,3.9,2.75,0.4139,1.95,13,0.415,2.9,0.447,5,0.401,1.5,2.5,0.575$, $0.75,0.421,2.45,0.41,8,1.35,0.4375,0.45,9.875,0.4155,1.3,0.41,0.44,0.4301,0.4325,2.2,0.402,0.411,0.75,1,0.65$, $0.435,0.42,0.4,0.401,6.083333,0.44,4,4.75,3.2,0.425,16.65,0.408,2.25,0.45,13.5,0.4,0.4159,1.2375,17,0.75$, $1.3,0.422,0.4,0.41,0.4,0.425,0.575,0.403,3.6,0.4125,0.4,0.475,3.75,3.375,0.5,0.407,4.25,0.4275,3.3,6.5,0.75,1$, $3.5,0.415,0.457,1.7,0.41,8,6.2,3.45,18.75,0.404,3.75,2.5,0.41,0.4017,3.06,15.5,0.44,4.5,12,7.666666,1.1,9.6$, $0.40807,1.85,1.3,3.75,3.275,0.525,0.405,0.455,0.40662,2.825,14,1,4,0.4,5.475,0.40175,0.41,10,0.44,0.4,11.4$ $, 0.4375,0.8,0.40125,0.43468,0.45,1,12.5,2.7,1.475,0.5,0.4,1.615,8.333666,10,0.4,0.425,0.4661,0.65,1,1,2.885$, $0.52,14.383049,0.405,1.15,0.46,0.401,0.555,15,6,1,1.8,2.75,1.15,0.404,0.4055,3.7,11.5,0.435,1,0.43,1.635$, $0.48,0.401,0.405,0.41,3.5,3.575,10.5,2,9.6,0.8,0.41,0.75,10,0.437,0.4,5,18.5,0.5,0.8,0.401,6.3,0.64,11.6,4.35$, $0.419,3.2,0.4,5.6,2.2,5,11.25,0.405,0.405,0.40473,0.415,12,2.8,0.4,0.44,15,9.5,0.65,1.4,0.4025,0.403,0.41$, $0.43,7.166666,7.75,12.868892,0.4,4.5,4.25,0.46,4,12,13.4,0.41482,0.4,2.65,0.4375,0.403,0.95,2.333333,2.3$, $0.41176,6.35,0.45,18.971596,10,0.44,6.5,1.4,12,11.5,0.4,0.4,5.775,0.435,12.083333,0.8225,0.41631,0.41,6.25$, $4.6,8.5,1.152,2.5,2.05,0.41089,0.4,0.55,2.095,15,1.15,8.5,0.413,0.401,7.166666,1.225,0.75,18,19.243682,4.25$, $0.405,1,0.405,0.405,7,1.6,6.25,0.475,2,1.85,4,13,2.7,0.401,2.625,1.625,0.455,13.054526,0.65,1.325,0.825$, $0.465,2.8,0.835,3.8,2.5,1.5,0.55,1.3,10,2,11.285714,0.4,0.6,0.445,10.125,2.275,0.405,12,3.125,0.405,2.4,8$ $, 0.4175,0.75,0.45,1.6125,2.5,7.666666,0.75,2.4,0.42,3.675,10.4,0.471,0.4,0.4,0.4087,0.42,12.125,10,1.1,2.6$, $0.4085,0.4,3.75,0.435,0.418,0.735,0.435,0.401,2.425,2.25,1.45,3.35,0.4,1.3,2,0.406,0.402,0.418,14.25,0.8$, $2.425,0.4,3.665,0.575,0.4015,0.425,2.59,8,5.375,0.4,1.1,0.4,2.15,0.42,0.4139,0.41,2,0.5,3.25,2.2375,2.2$, $12.25,0.64,0.85,0.4,3.5,3.8,12,0.4015,18,0.4118,8,14,0.4,0.475,6,2.3,0.5,0.4144,0.41,8.5,1.9,5,2.25,0.825$, $0.4,0.4052,2.4,0.4,0.42,4.75,0.925,1.5,9.85,1.9,2.75,0.4,0.95,0.465,6.125,0.4,2.825,0.4194,0.4,1.5,2.25,1$ ,9.166666, $0.4015,0.4,0.85,5,0.418,1.4,0.475,18.876139,7.05,13.302583,7.95,4.5,0.42,0.66,5,4.9,0.4135,0.825$, $0.4037,2.5,5.1875,0.41,1.825,14,2.5,2.5,0.4023,1.5,2,0.4,0.405,0.4095,2.5,0.4,6.4,0.5,2.25,0.4,3.1,1.7,0.75$ $, 0.4,11.625,7.8,0.44,2.4625,7.5,10.5,0.4115,12.137,0.4,11.166666,0.4375,2.4,3.364877,7.75,0.4145,6.5,12,0.4$, $3.5,0.4125,0.467,0.403075,0.4135,0.405,1.1,0.476,0.4161,0.44,0.404,1.25,6.25,0.95,0.4014,14,3.35,0.4,0.437$, $16.5,3.2,7.4375,1.015,0.4495,0.4167,0.404,12.433333,1.4,1.875,3.7,4.6875,0.4,2.9375,0.75,0.465,6,0.4,0.411$, $1.6,0.55,2.5,5.5,1.25,0.432575,7.4,0.5,2,1.5,0.4203,2.225,3.9,0.4033,1.3,3.3125,1.9,0.4,0.4,0.4,0.40075,13$, $0.4148,0.4,2.6,1,5.5,5.35,2.35,0.414,0.4299,7.5,0.431,1.35,4,0.4,10,21.6,1.255,14.427326,0.4155,12.5,0.4214$, $0.4,23.854494,3.75,0.75,0.4,8,0.414,0.4461,1.55,15.217401,13,0.4,1,0.4155,9.25,11.5,14.5,0.401,2.125,0.75$, $0.403,0.4,0.4155,0.4038,1.25,0.4,6.55,0.4,0.43,8,8.333333,3,0.75,0.4025,0.4,0.85,0.65,0.42$.

On the basis of the two data sets, we compare fits from the ETLD with those of its sub-models, namely, exponentiated (generalized)Lindley distribution (ELD), transmuted Lindley distribution (TLD) and Lindley distribution (LD). Estimates of all the model parameters are found through the maximum likelihood procedure. The criteria used for this comparison are the AIC and BIC. Given the sample size n, number of parameters contained in a model $k$ and estimate of the log-likelihood function $(L L)$ which corresponds to the maximum likelihood estimates, these criteria are defined as follows:

$$
A I C=-2 L L+2 k \text { and } B I C=-2 L L+k \ln (n) .
$$

A distribution is said to provide the best fit to the data if among all the distributions under consideration, it corresponds to minimum values of AIC and BIC. Maximum likelihood estimates, standard error estimates and values of the criteria are given in Table 2 for the selected distributions and first data.

Results in Table 2 indicate that ETLD corresponds to the smallest AIC and BIC values, making it the best among all the distributions which have been fitted to the data.

For the second data, the maximum likelihood estimates of the model parameters, their respective standard errors and AIC and BIC values are reported in Table 3.

Table 3 clearly shows that the ETLD has the minimum values of AIC and BIC among the fitted distributions. Thus, the ETL distribution is the best model for modelling the data among the distributions considered in Table 3. 
Table 2. Parameter Estimates and Corresponding Values of Model Selection Criteria for the Distributions Fitted to Data 1

\begin{tabular}{||cccccc||}
\hline Distribution & Estimate & Standard Error & LL & AIC & BIC \\
\hline \hline $\operatorname{ETL}(\alpha, \lambda, \theta)$ & $\hat{\alpha}=0.7079$ & 0.0726 & 245.7743 & -485.5485 & -476.2126 \\
& $\hat{\lambda}=0.4868$ & 0.1888 & & & \\
& $\hat{\theta}=7.5782$ & 1.1301 & & & \\
\hline $\operatorname{EL}(\alpha, \theta)$ & $\hat{\alpha}=0.6389$ & 0.0615 & 242.8272 & -481.6544 & -475.4304 \\
& $\hat{\theta}=8.8049$ & 0.9470 & & & \\
\hline $\operatorname{TL}(\lambda, \theta)$ & $\hat{\lambda}=0.5554$ & 0.1249 & 239.783 & -475.5659 & -469.3419 \\
& $\hat{\theta}=9.4377$ & 0.9613 & & & \\
\hline $\operatorname{L}(\theta)$ & $\hat{\theta}=11.8196$ & 0.8555 & 230.9877 & -459.9754 & -456.8634 \\
\hline
\end{tabular}
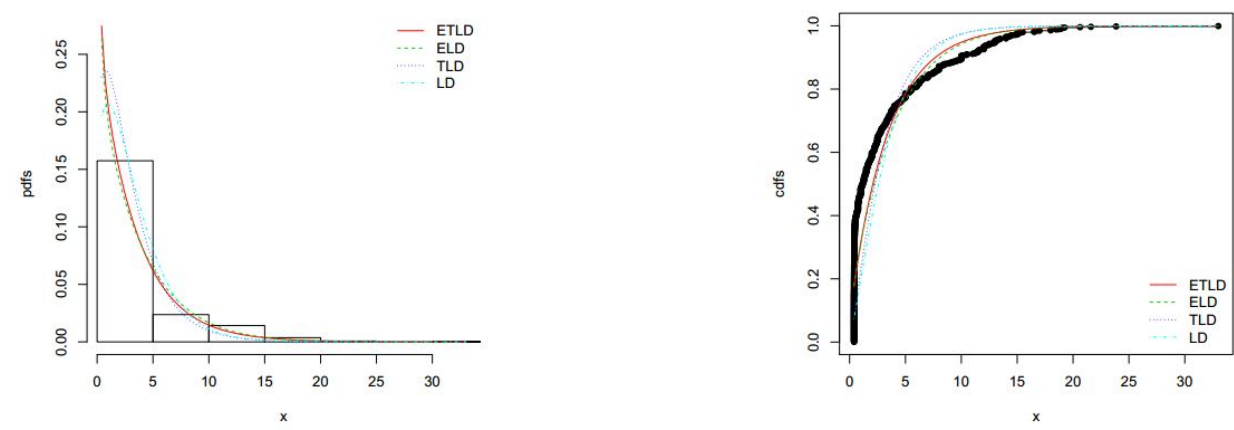

Figure 17. Graphs of the estimated pdfs and cdfs based on the first data

Table 3. Parameter Estimates and Corresponding Values of Model Selection Criteria for the Distributions Fitted to Data 2

\begin{tabular}{||cccccc||}
\hline Distribution & Estimate & Standard Error & -LL & AIC & BIC \\
\hline \hline $\operatorname{ETL}(\alpha, \lambda, \theta)$ & $\hat{\alpha}=0.5820$ & 0.0281 & 1806.964 & 3619.928 & 3634.0419 \\
& $\hat{\lambda}=0.3918$ & 0.0792 & & & \\
& $\hat{\theta}=0.3264$ & 0.0170 & & & \\
\hline $\operatorname{EL}(\alpha, \theta)$ & $\hat{\alpha}=0.5396$ & 0.0246 & 1818.004 & 3640.008 & 3649.422 \\
& $\hat{\theta}=0.3546$ & 0.0151 & & & \\
\hline $\operatorname{TL}(\lambda, \theta)$ & $\hat{\lambda}=0.5022$ & 0.0482 & 1874.227 & 3752.453 & 3761.867 \\
& $\hat{\theta}=0.4432$ & 0.0138 & & & \\
\hline $\operatorname{L}(\theta)$ & $\hat{\theta} 2=0.5099$ & 0.0130 & 1919.938 & 3841.876 & 3846.583 \\
\hline
\end{tabular}

\section{Conclusion}

In as much as the quality of the empirical results obtained by applying many parametric methods of analysis greatly depends on how well the chosen distribution fits the data under consideration, efforts will always be made to generalize distributions. We have introduced and studied the properties of a new distribution called the exponentiated transmuted Lindley distribution. Specifically, we have derived the quantile function, the expression for the raw moments, moment generating function and the pdf of the $k t h$ order statistic based on the distribution. Through a numerical illustration, the distribution is found to be capable of being positively skewed and platykurtic or positively skewed and leptokurtic. Under several conditions, the relationships between each of the mean, median, variance, skewness and kurtosis of the distribution and the parameters are studied. In particular, when only two parameters are constant, it is interesting to know that none of skewness and kurtosis is a linear function of the other parameter. With 

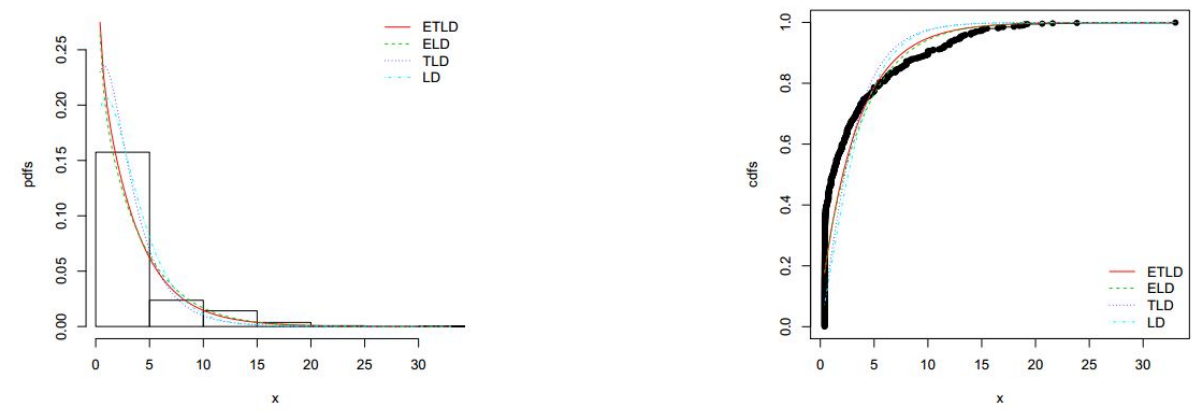

Figure 18. Graphs of the estimated pdfs and cdfs based on the second data

two data sets, the applicability as well as the ability of this distribution to outperform its sub-models in many data analysis situations is illustrated. Therefore, the model is recommended for modelling right-skewed data.

Conflicts of Interest: "The author declare no conflict of interest."

\section{References}

[1] Ghitany, M. E., Atieh, B., \& Nadarajah, S. (2008). Lindley distribution and its application. Mathematics and computers in simulation, 78(4), 493-506.

[2] Bakouch, H. S., \& Popovi', B. V. (2016). Lindley first-order autoregressive model with applications. Communications in Statistics-Theory and Methods, 45(17), 4988-5006.

[3] Fatima, A., \& Roohi, A. (2015). Transmuted exponentiated pareto-i distribution. Pakistan Journal of Statistics, 32(1), 63-80.

[4] Nadarajah, S., Bakouch, H. S., \& Tahmasbi, R. (2011). A generalized Lindley distribution. Sankhya B, 73(2), 331-359.

[5] Merovci, F. (2013). Transmuted lindley distribution. International Journal of Open Problems in Computer Science and Mathematics, 238(1393), 1-20.

[6] Mansour, M. M., \& Mohamed, S. M. (2015). A new generalized of transmuted Lindley distribution. Appl. Math. Sci, 9, 2729-2748.

[7] Kemaloglu, S. A., \& Yilmaz, M. (2017). Transmuted two-parameter Lindley distribution. Communications in Statistics-Theory and Methods, 46(23), 11866-11879.

[8] Granzotto, D. C., Mazucheli, J., \& Louzada, F. (2016). Statistical study of monthly rainfall trends by using the transmuted power Lindley distribution. International Journal of Statistics and Probability, 6(1), 111-124.

[9] Nofal, Z. M., \& Abd El Hadi, N. E. (2015). Exponentiated transmuted generalized Raleigh distribution: A new four parameter Rayleigh distribution. Pakistan journal of statistics and operation research, 11(1), 115-134.

[10] Fattah, A. A., Nadarajah, S., \& Ahmed, A. H. N. (2017). The exponentiated transmuted Weibull geometric distribution with application in survival analysis. Communications in Statistics-Simulation and Computation, 46(6), 4244-4263.

[11] Pal, M., \& Tiensuwan, M. (2015). Exponentiated transmuted modified Weibull distribution. European Journal of Pure and Applied Mathematics, 8(1), 1-14.

[12] Kenney, J. F. (2013). Mathematics of statistics. D. Van Nostrand Company Inc.

[13] Moors, J. J. A. (1988). A quantile alternative for kurtosis. Journal of the Royal Statistical Society: Series D (The Statistician), $37(1), 25-32$.

[14] Smithson, M., \& Verkuilen, J. (2006). A better lemon squeezer? Maximum-likelihood regression with beta-distributed dependent variables. Psychological methods, 11(1), 54-71.

[15] Bourguignon, M., Ghosh, I., \& Cordeiro, G. M. (2016). General results for the transmuted family of distributions and new models. Journal of Probability and Statistics, 2016, 1-12.

[16] Oluyede, B. O., \& Rajasooriya, S. (2013). The Mc-Dagum distribution and its statistical properties with applications. Asian Journal of Mathematics and Applications, 2013, 1-16. 
(C) 2019 by the authors; licensee PSRP, Lahore, Pakistan. This article is an open access article distributed under the terms and conditions of the Creative Commons Attribution (CC-BY) license (http://creativecommons.org/licenses/by/4.0/). 